\title{
WFIRST CGI integral field spectrograph performance and post- processing in the OS6 observing scenario
}

Maxime J. Rizzo, Neil T. Zimmerman, Tyler D. Groff, John Krist, A.J. Eldorado Riggs, et al.

Maxime J. Rizzo, Neil T. Zimmerman, Tyler D. Groff, John Krist, A.J. Eldorado Riggs, Qian Gong, Avi M. Mandell, Prabal Saxena, Michael W. McElwain, Aki Roberge, "WFIRST CGI integral field spectrograph performance and postprocessing in the OS6 observing scenario," Proc. SPIE 10698, Space Telescopes and Instrumentation 2018: Optical, Infrared, and Millimeter Wave, 106986U (1 August 2018); doi: 10.1117/12.2312400

Event: SPIE Astronomical Telescopes + Instrumentation, 2018, Austin, Texas, United States 


\title{
WFIRST Coronagraph Integral Field Spectrograph Performance in the OS6 Observing Scenario
}

\author{
Maxime J. Rizzo ${ }^{a, c}$, Neil T. Zimmerman ${ }^{a}$, Tyler D. Groff ${ }^{a}$, John Krist $^{b}$, A J Eldorado Riggs ${ }^{b}$, \\ Qian Gong $^{a}$, Avi M. Mandell ${ }^{a}$, Prabal Saxena ${ }^{a, c}$, Michael W. McElwain ${ }^{a}$, Aki Roberge ${ }^{a}$ \\ ${ }^{a}$ NASA Goddard Space Flight Center, 8800 Greenbelt Road, Greenbelt, MD, 20771, USA \\ ${ }^{b}$ Jet Propulsion Laboratory, California Institute of Technology, Pasadena, CA, USA \\ ${ }^{c}$ NASA Postdoctoral Fellow
}

\begin{abstract}
The WFIRST coronagraph instrument (CGI) will have an integral field spectrograph (IFS) backend to disperse the entire field of view at once and obtain spatially-resolved, low-resolution spectra of the speckles and science scene. The IFS will be key to understanding the spectral nature of the speckles, obtain science spectra of planets and disks, and will be used for broadband wavefront control. In order to characterize, predict, and optimize the performance of the instrument, we present a detailed model of the IFS in the context of the new OS6 observing scenario. The simulation includes spatial, spectral, and temporal variations of the speckle field on the IFS detector plane, which allows us to explore several post-processing methods and assess what gains can be expected. The simulator includes the latest models of the detector behavior when operating in photon-counting mode.
\end{abstract}

Keywords: Integral field spectroscopy, coronagraphy, high-contrast, spectrograph

\section{INTRODUCTION}

WFIRST CGI is a technology demonstration that will pioneer active wavefront control in high-contrast coronagraphy from space. Through its state-of-the-art architecture, coronagraph mask design and wavefront control algorithms, CGI will reach starlight suppression levels on the order of $\sim 10^{-9}$ at visible wavelengths - orders of magnitude more than what can currently be achieved from the best ground-based facilities. This new region of phase space opens access to new astronomical objects of interest such as cold giant planets orbiting nearby Sun-like stars, and will give us the opportunity to peak at their atmospheric composition.

CGI will feature an Integral Field Spectrograph (see Fig. 1) as one of its two focal plane instruments, the other being a direct imager. The IFS design is inspired from integral field spectrographs deployed at ground-based observing facilities and tailored for visible light. PISCES, ${ }^{1-3}$ a prototype IFS, is currently installed at JPL's High Contrast Imaging Testbed (HCIT) and has reached contrasts close to $1 \times 10^{-8}$ in full $18 \%$ bandpasses in the laboratory, a result that is not limited by the performance of the spectrograph. Through 2018 and into 2019, PISCES will be installed on a more flight-like chamber and is set to reach two or three times deeper contrast in the presence of dynamic perturbations. The parameters and layout of the flight IFS can be found in a companion paper by Groff et al. in these proceedings.

CGI has entered Phase B in June 2019 and is now refining its design. This effort is centered on increased modeling fidelity of the instrument's behavior and a refinement of the design reference mission or observing scenario.

In order to support this modeling effort, we have developed a comprehensive software package in Python, crispy, ${ }^{4}$ that is used to simulate CGI and CGI-IFS data products for use by the science teams and to determine instrumental sensitivities on useful science metrics. This is particularly important for coronagraphs, which cannot rely solely on analytical imaging metrics such as the full-width at half-maximum, but instead rely on the performance of post-processing algorithms to increase the final signal-to-noise ratio (SNR).

Send correspondence to: maxime.j.rizzo@nasa.gov

Space Telescopes and Instrumentation 2018: Optical, Infrared, and Millimeter Wave, edited by Makenzie Lystrup,

Howard A. MacEwen, Giovanni G. Fazio, Proc. of SPIE Vol. 10698, 106986 U

(C) 2018 SPIE · CCC code: $0277-786 X / 18 / \$ 18 \cdot$ doi: $10.1117 / 12.2312400$

Proc. of SPIE Vol. 10698 106986U-1 


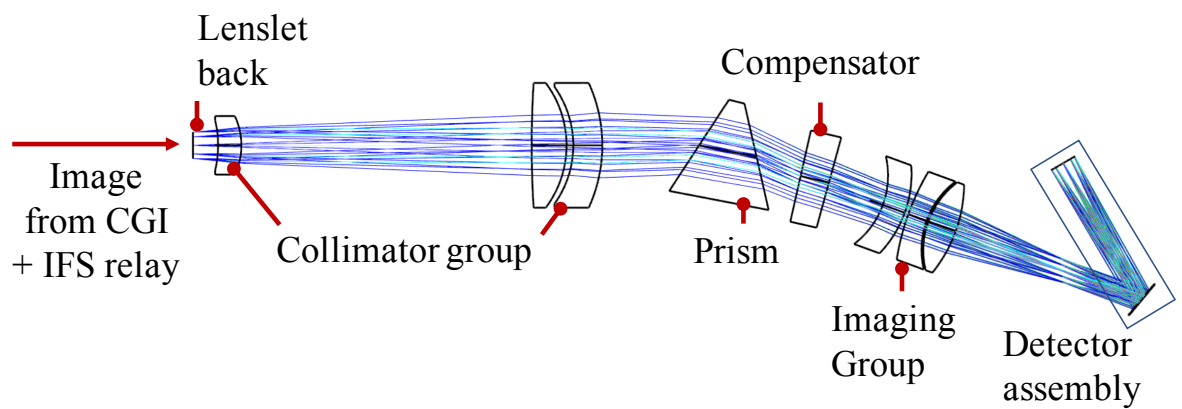

Figure 1. WFIRST flight Integral field spectrograph layout.

In this paper, we first discuss improvements made to crispy which increase its robustness, flexibility, and general usefulness. Second, we discuss the characteristics of the new WFIRST observing scenario, labeled OS6. Third, we show the results of the newly-implemented crispy capabilities in processing pure coronagraph pointspread functions (PSF) and creating useful CGI data products with post-processing. Finally, we show the improvements in spectroscopic simulation capabilities of crispy in the OS6 scenario.

\section{SOFTWARE IMPROVEMENTS}

The general outline of crispy is described in Rizzo et al. (2017), ${ }^{4}$ and has not significantly changed (see an outline in Fig. 2). We recommend the reader to read that publication prior to reading this one, as a lot of the features of crispy are not described again here. In the past year crispy has been expanded to include both IFS and the direct imager simulations and post-processing. It relies on ingesting a time series of resolved coronagraph PSFs, and allows the propagation of a full science scene on the Direct Imaging Camera or the IFS (see Section 3). It also includes several post-processing routines that allows the user to eliminate systematic noise terms in the CGI images and increase the planet SNR.

One notable improvement in crispy is the extraction algorithm, which converts the detector maps back into spectral cubes. We have establish a novel extraction method that optimizes the operation in the presence of Poisson statistics, which will be discussed in a subsequent publication (Rizzo et al. 2018, in prep.).

\subsection{Brief summary of crispy's architecture}

The main role of the simulation software is to propagate wavelength-resolved focal plane cubes onto a 2D detector pixel array. The architecture is designed to conserve photon fluxes but takes into account detector quantum efficiency during this propagation step. Realistic detector noise, including electron-multiplying gain register noise, can be added to the 2D maps to produce realistic data products. Finally, an extraction routine is used to extract the information from the $2 \mathrm{D}$ detector map back into a 3D cube representing the input scene convolved by the spectrograph's spatial and spectral response function. This extraction step is currently implemented on the PISCES testbed and is functioning well on lab data.

\subsection{Wavelength calibration improvement}

The CGI IFS requires an absolute wavelength accuracy of $2 \mathrm{~nm}$ when extracting spectra, which puts some constraints on the system stability since no on-board calibration source is currently baselined in the instrument. We used the crispy wavelength calibration module on the PISCES IFS data, and we observed a significant amount of residual flux post-spectral extraction, which indicates a registration error in the wavelength calibration step. crispy was originally based on the CHARIS data reduction pipeline ${ }^{5}$ which uses low-order polynomials to fit grids of monochromatic PSFlets across the detector. This is usually enough to fit the overall grid in the non-telecentric system. If these PSFlets are correctly arranged on a smoothly varying grid, this method has very high SNR and results in a wavelength solution which is much better the the SNR of each individual PSFlet. This is because the thousands of PSFlets are all used together to fit only $\sim 20$ polynomial parameters. 


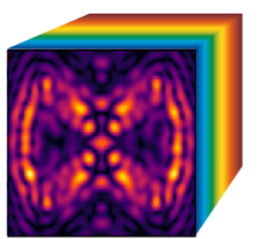

Wavelength-resolved Input cubes

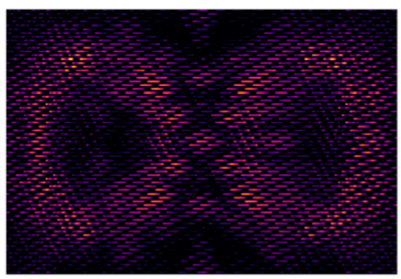

IFS detector photon flux map

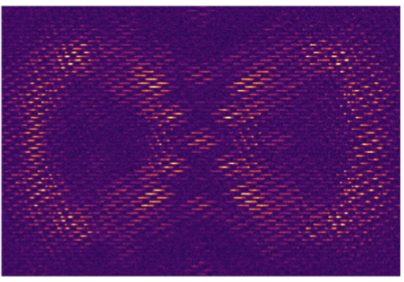

IFS detector electron map

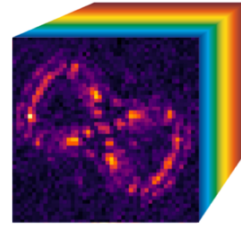

Extracted cubes

Figure 2. crispy can both simulate IFS focal plane maps and extract 2D detector maps back into 3D spatio-spectral cubes. The maps can be modeled with realistic detector noise in order to produce realistic data products.

However, high-frequency spatial variations in the manufacturing of the lenslet array (as observed in PISCES) can make fitting monochromatic flatfields to a smoothly-varying grid non-optimal. These variations result in an error in each position of the PSFlet with respect to the smooth linear fit, which was observed in the PISCES lab data. Because $2 \mathrm{~nm} \simeq 0.3$ pixel along the dispersion direction on the detector, it is important to increase the robustness of the wavelength calibration method to ensure that we can get that accuracy for each individual PSFlet or microspectrum.
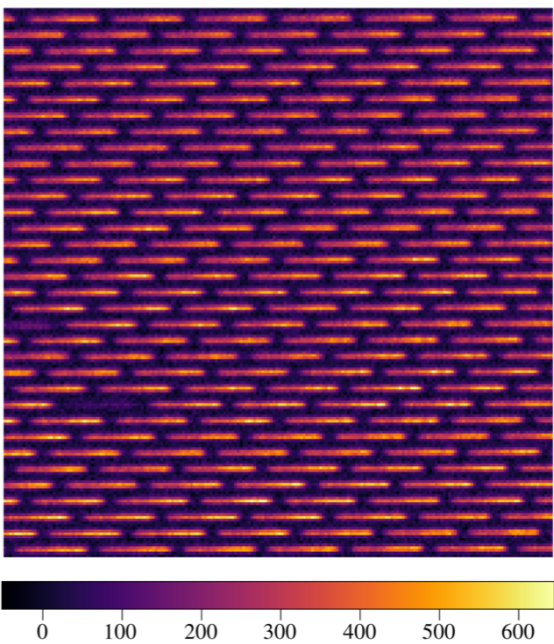

(a) Original PISCES broadband flatfield
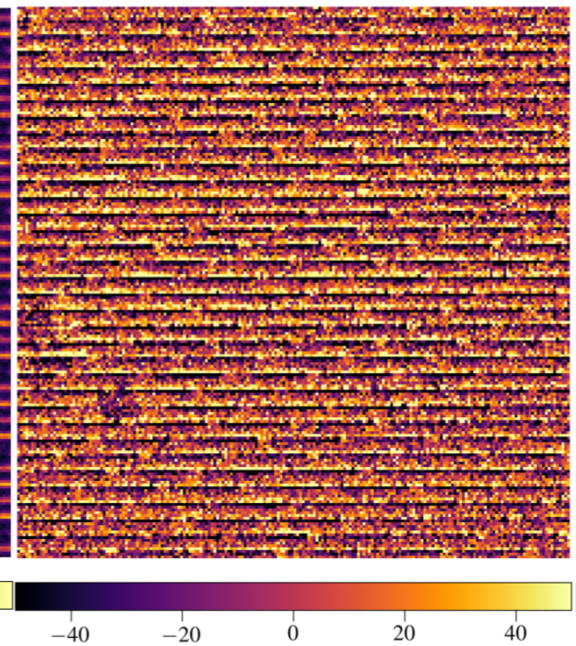

(b) Residuals with polynomial wavelength calibration

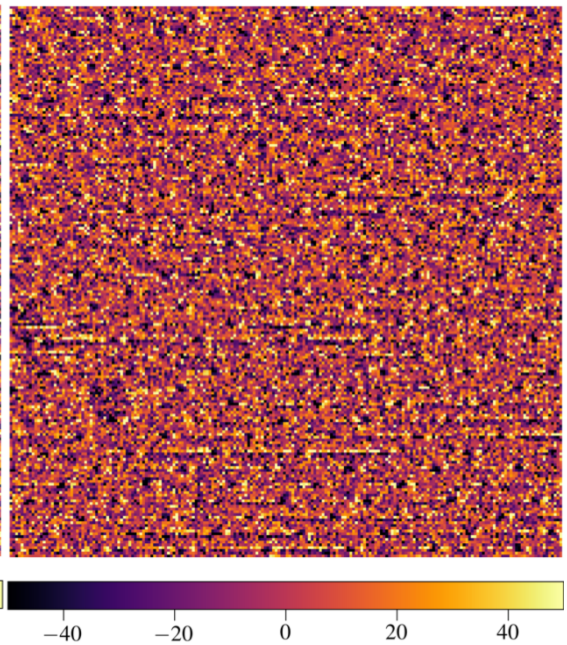

(c) Residuals with fine wavelength calibration

Figure 3. crispy has improved the wavelength calibration methods over the CHARIS-style polyomial fitting. The new method is particularly well suited for PISCES where errors in the regularity of the lenslet array need to be accounted for. (a) shows an original broadband flatfield from the PISCES IFS at HCIT, which is already dark-subtracted. (b) Shows the residuals after a least squares extraction method using psflet registration and wavelength calibration established using a global, low-order polynomial fit on monochromatic fields. A systematic residual is present, which indicates that we are missing flux when fitting out the microspectra, and we could be off in our absolute wavelength registration. (c) Shows the improved residuals after applying our fine calibration method. In this method, each microspectra is registered independently with several high SNR fields. Very few systematic signatures remain.

We implemented a fine calibration method which determines an offset vector $[d x, d y]^{T}$ in pixels for each lenslet. This offset is with respect to the smooth 2-D polynomial fit. The implementation is simple: we first fit the rough position of the PSFlet with the regular polynomial fit for every monochromatic wavelength in our calibration dataset, then apply a centroiding algorithm for each PSFlet individually. These offsets are thus recorded and averaged over all wavelengths, with the assumptions that they are static and do not change between 
the times the various monochromatic wavelength maps were obtained. These offsets are then added to the global positions of the PSFlets during the wavelength extraction step. We show that the error on the lenslet position along the dispersion direction is $\sigma_{x} \simeq \mathrm{SNR}^{-1}$ for a critically sampled PSFlet of 2 pixels per FWHM. As expected, this is improved by $\sqrt{N}_{\lambda}$ when averaging $N_{\lambda}$ wavelengths. In Fig. 3, we show the residual of a least squares extraction without and with the fine calibration method for PISCES.

\subsection{Detector model improvements}

The latest version of crispy augments the 2017 EMCCD model with a cosmic ray and hot pixel simulator developed by P. Morrissey (private communication). The code uses models of cosmic ray tails and hot pixel maps derived from empirical lab measurements at JPL. In Fig. 4 we show an example of a CGI frame of 60 seconds with the cosmic ray tails clearly visible, which are along the serial register readout direction. These models are simplified for now and have several limitations. First, the cosmic ray tails always have the same profile; second, the models are only representative of hits on single pixels, not multiple pixels; third, the digitization of the detector maps is still done with 16 bits, while the flight detector electronics will only have 14 bits; and fourth, the models still do not correctly model the charge traps behavior accurately.

These approximations could make the current detector model somewhat optimistic compared to the final performance during flight, but CGI is also adding features to the CCD that will help mitigate some of these effects (see Morrissey et al., 2018, in these proceedings).

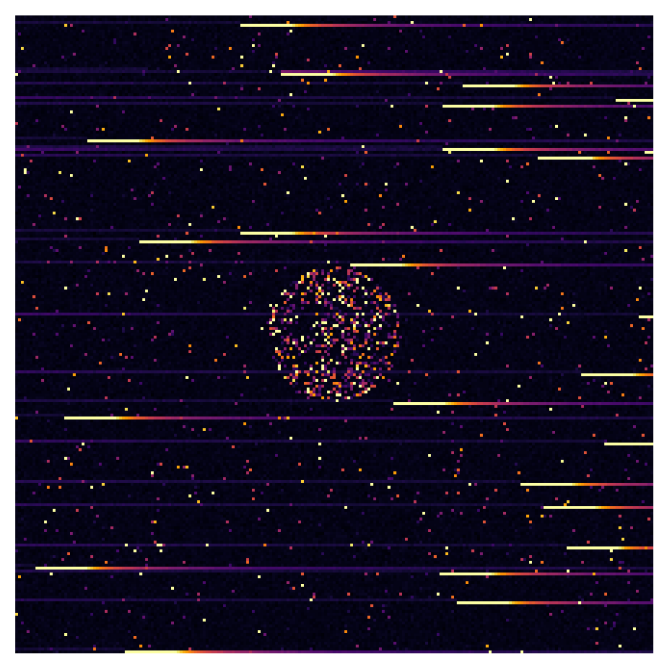

Figure 4. Single exposure of $60 \mathrm{~s}$ with the WFIRST detector model, including cosmic rays and hot pixels. The circle towards the center of the image corresponds to the HLC mask size an is about 45 pixels wide.

The code for the detector simulation will be made public on the GitHub repository after it has gone through the JPL code release system.

\section{CGI RESULTS WITH OS6}

In this paper we showcase the usefulness of crispy by simulating an entire OS6 observing sequence. The coronagraphic PSFs are simulated by J. Krist and are available on the WFIRST website*. The simulation of these PSFs, from the observatory structure, thermal and optical model down to the closed-loop coronagraphic

\footnotetext{
*https://wfirst.ipac.caltech.edu/https://wfirst.ipac.caltech.edu/
} 
operation is handled by JPL (Krist et al., 2018, these proceedings). Two cases are simulated, one corresponding to the coronagraph best performance estimates, and one corresponding to the performance when accounting for margin uncertainty factors (MUFs).

The design reference mission for the coronagraph instrument in OS6 is a series of target observations chopped every $\sim 10$ hours with a slew to a reference star. The target is observed during $\sim 8$ hours in two pairs of roll positions ( $\sim 2$ hours each), and the reference is observed for $\sim 2$ hours (Fig. 5$)$. The cycle of $\sim 10$ hours is repeated 13 times. The data products have a time resolution of 120 seconds, which is the maximum exposure time of an image on-orbit, limited by cosmic ray contamination.

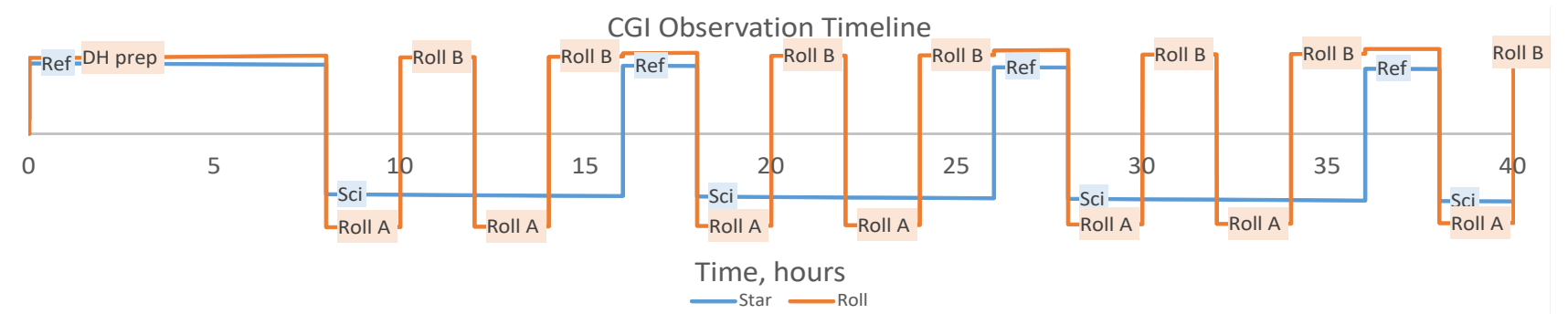

Figure 5. OS6 timeline and observing sequence showing the chopping scheme alternating rolls and reference star observations (Nemati et al., 2018, these proceedings).

crispy ingests these flux maps and creates realistic data products with all detector noise sources, after propagating a full 2-dimensional science scene. In addition, crispy implements several post-processing routines such as traditional least squares reference differential imaging (RDI), or principal component analysis (PCA) based methods such as KLIP. ${ }^{6}$

\subsection{OS6 correlation matrix}

To take a look at the global OS6 data, we average images in sets of 2 hours, and measure the cross-correlation between all 2-hour averages. The correlation matrix (Fig. 6) shows the results for the OS6 HLC simulation with MUFs, with no noise terms (infinite SNR). Every pixel corresponds to the correlation coefficient between two sets of averaged 2-hour simulated data. It indicates that the correlation between noiseless images is maintained very high throughout the entire OS6 observing sequence, which is what is desired to maximize the effectiveness of post-processing strategies. Every 20 hour or so, a spike in contrast (due to high jitter values) corrupts the averages which leads to slightly lower correlation values. An effort will be made to identify the images with worse behavior and eliminate them from the global average.

In Fig. 6 (b), we show the correlation matrix with MUFs and with detector noise, without the cosmic rays for simplicity. Here the repointings to the reference star are more obvious with higher correlation, which is because they have more SNR. A lot of the low-level speckle pattern in the dark hole now have much lower SNR in the target images. The high-jitter cases are still visible, repeating about every 20 hours or so (10 pixels on the matrix).

\subsection{Input maps}

We use the Haystacks software ${ }^{7}$ to create representative scenes adapted to the WFIRST CGI. In this case, we construct a dust model from C. Stark ${ }^{8}$ that is dynamically consistent with a 1 Jupiter mass planet at 1 astronomical unit from its host. We scale the dust to have a surface brightness of about 1.5 zodi at the peak of the emission (with 1 zodi defined as $4 \times 10^{-6} \mathrm{Jy} / \mathrm{asec}^{2}$ ). The planet in the input maps shown in Fig. 7 is at $6 \times 10^{-9}$, which is fainter than the CGI HLC requirements. The central star corresponds to a $\mathrm{V}=5$ mag star.

\subsection{Post-processing}

Result of the OS6 stack and a KLIP algorithm on the data are shown in Fig. 8. These images incorporate cosmic ray models, but the cosmic rays have been flagged and the entire rows have been masked out during the averaging. With this current cosmic ray model, the existence of cosmic rays simply affects the overall 


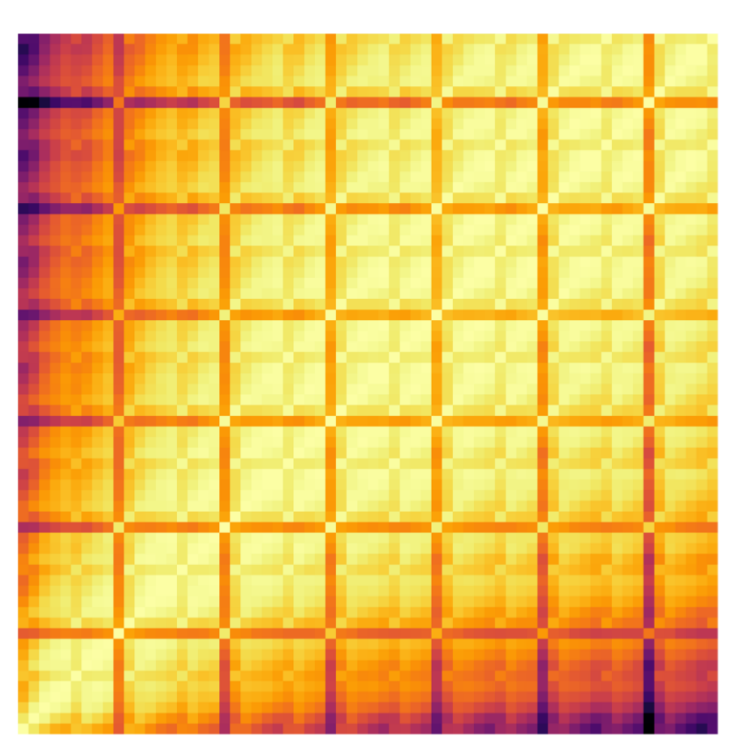

(a) Noiseless images

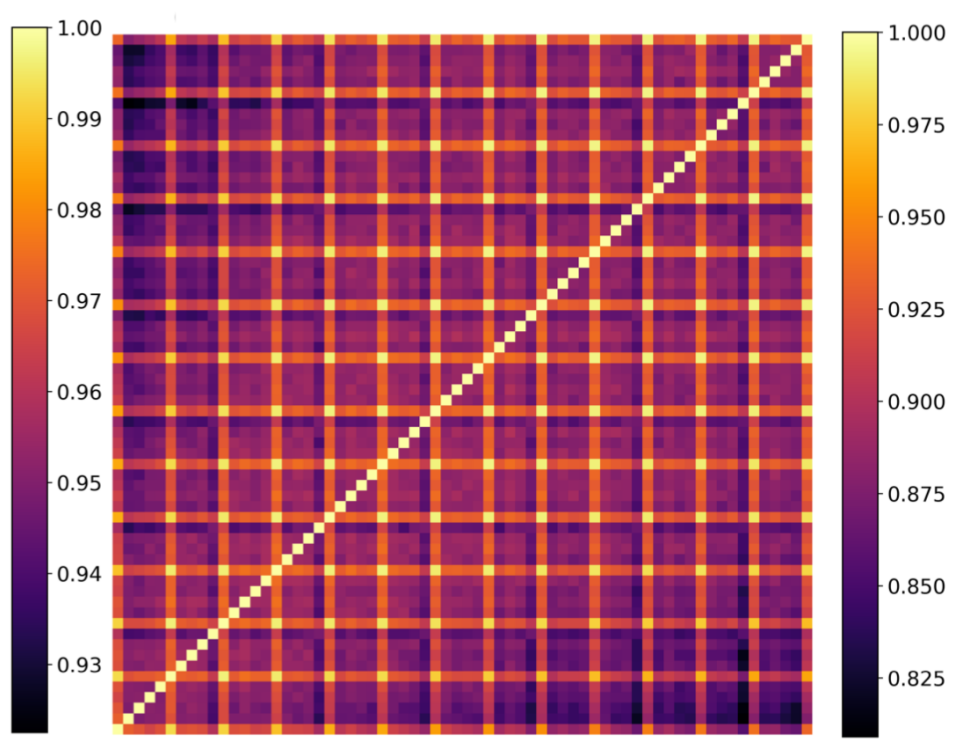

(b) Noisy images

Figure 6. Data correlation between 2-hour chunk averages for OS6. Every pixel of this matrix represents the correlation coefficient between two 2-hour averages. (a) Shows the correlation for noiseless images (raw OS6 as present on the website). The periodic grid of lower correlation every $\sim 20$ hours or so corresponds to high jitter phases where the dark hole is corrupted. No attempt is made to remove these frames, as this is just processing the raw data. (b) Shows the same correlation but now for images with realistic exposure times and detector model (although the cosmic rays have been omitted for now, as it just reduced overall QE). The periodic pattern of high correlations corresponds to the revisits to the reference star. A relatively high correlation coefficient is maintained throughout the entire sequence, which will maximize the effectiveness of the reference differential imaging methods.

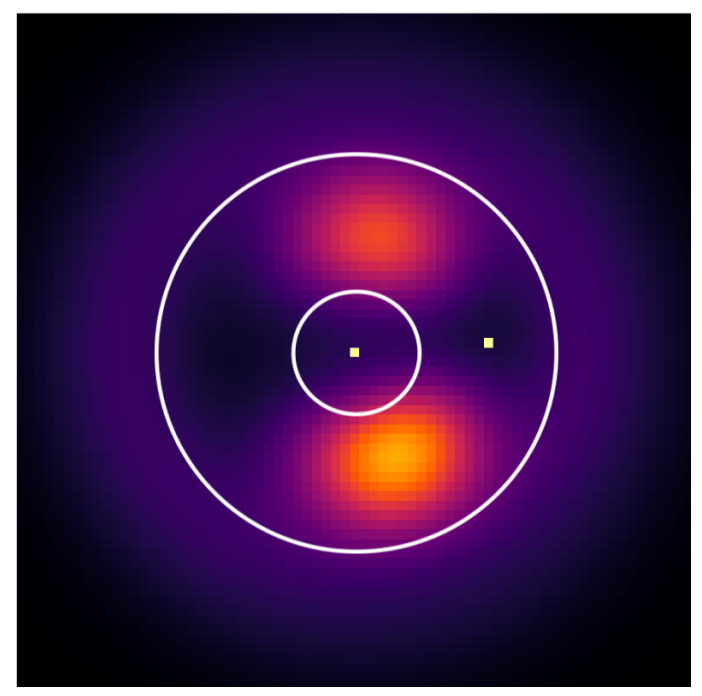

Figure 7. Input flux map, with the HLC coronagraph inner and outer working angles outlined in white for reference. The planet in this image is at a contrast of $6 \times 10^{-9}$, and the dust peaks at about $6 \times 10^{-6} \mathrm{Jy} / \mathrm{asec}^{2}$.

efficiency of the observation, and does add a slight bias to the real incoming flux on the overall image during contrast normalization. We expect these effects can be accounted for with future investigations in the detector systematics.

The KLIP method is described in detail in Pueyo et al, 2016, ${ }^{6}$ but is implemented simply in the crispy tools. In essence, the algorithm uses a singular value decomposition approach of the ensemble of 2-hour reference sets, and keeps only the eigenvectors with the highest eigenvalues. For each set of target observations, individual 
frames are averaged, then projected on this new basis of eigenvectors. The model obtained is then subtracted and the residual shows only the non-common structure, such as the dust and the planet. In Fig. 8 we show the post-processed results for both roll angles. Increased SNR can be obtained by combining the two rolls.
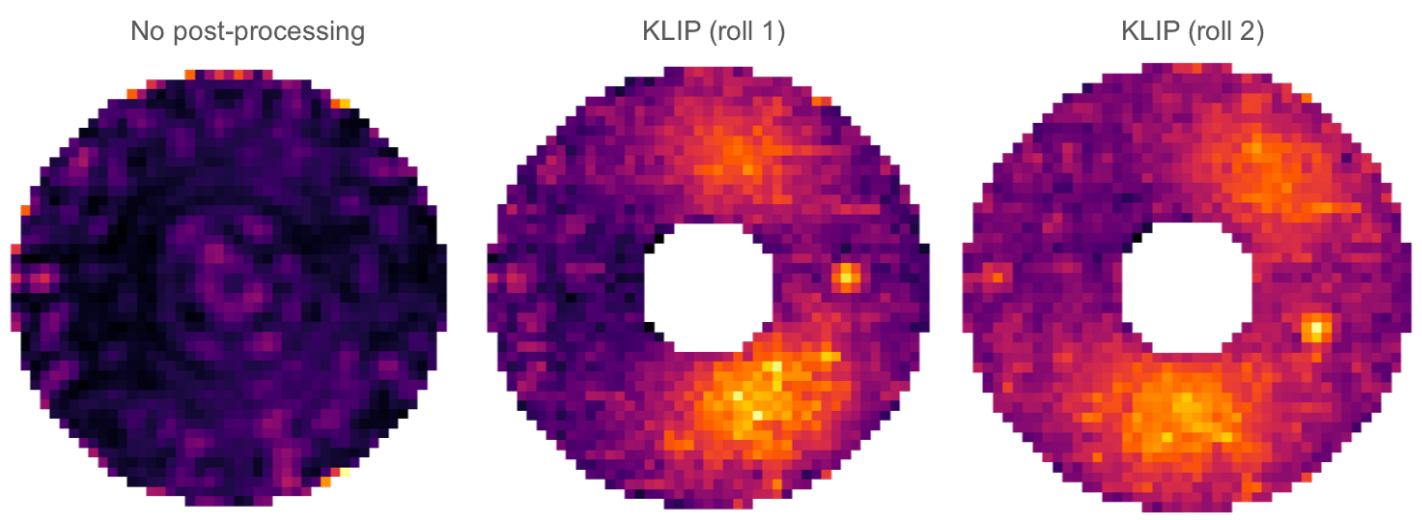

Figure 8. Example of post-processing results with the OS6 datafiles. Left: stacked target star for Roll 1 without any post-processing; Middle: After KLIP for Roll 1; Right: After KLIP for Roll 2. In the post-process data, both the planet and the resonant dust structure can be recognized. Note that the long duration of the OS6 simulation ( $\sim 130$ hours) is longer than what would be typically spent on targets like these, so the fact that we detect the planet with high SNR is expected.

\section{SPECTROGRAPH RESULTS WITH OS6}

A similar approach is used to simulate IFS data products using OS6, for which spectrally, spatially and temporally resolved focal plane maps are provided for the main IFS band centered at $770 \mathrm{~nm}$. Because the field of view of the Shaped Pupil bowtie mask is small, we do not propagate an entire scene through the IFS, but instead only a single off-axis point source. In addition, because the bowtie has a very narrow opening angle and the PSF is wide, we do not use any rolling of the spacecraft. While OS6 implements rolling no matter what, we choose to propagate the planet at the same location in both rolls. An example of a propagated map is shown in Fig. 9.

\subsection{Cosmic rays}

Cosmic rays pose a major problem for the IFS maps. Because the IFS requires much more pixel real estate than the direct imager, cosmic rays will be much more likely to affect the signal. Because the IFS microspectra are aligned along the pixels of the detector, a single cosmic ray with a tail along the same direction can contaminate all wavelengths for many microspectra. We thus recommend that the serial register direction (which determines the direction of the cosmic ray tails) be perpendicular to the IFS microspectra direction when possible. This way, a cosmic ray tail will impact discrete wavelengths for multiple lenslets, but will not completely prevent the retrieval of the signal for many microspectra.

\subsection{Post-processing and extraction}

Below we show the results from two simulation cases. The first one represents the results with a detector model at the beginning of life, without hot pixels or cosmic rays (Fig. 10); the second one represents the results with a detector model at 3 year lifetime, with hot pixels and cosmic rays (Fig. 11). The cosmic rays are removed following the same method as for the HLC simulations (the corresponding rows are just ignored). 


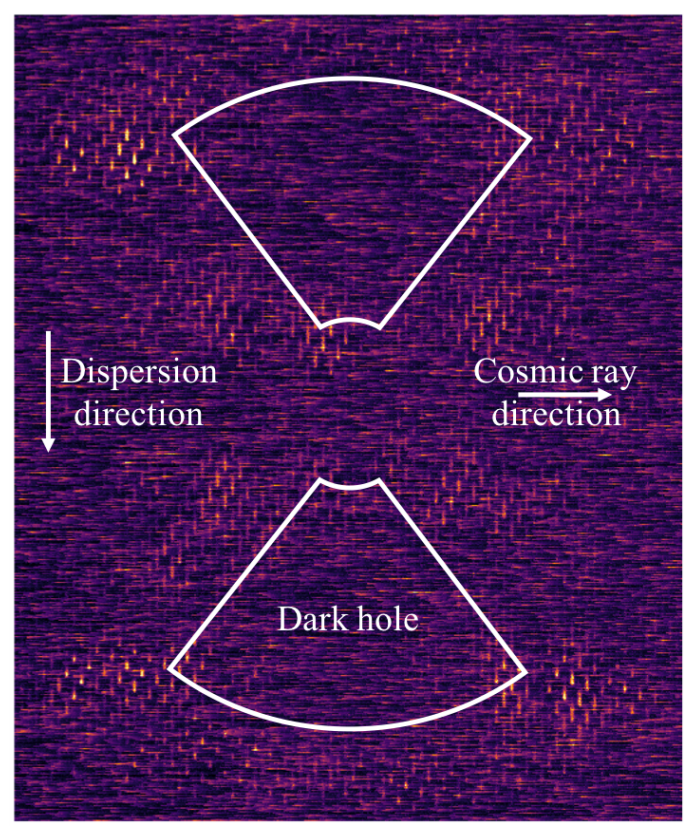

Figure 9. Average of 2-hour reference star IFS observation, at 3 year lifetime with cosmic rays and hot pixels. In this image, the average is done without any attempt to remove cosmic rays. The orientation of the cosmic ray tails is perpendicular to the dispersion direction. The approximate region of the dark hole is indicated by the bowtie shape.

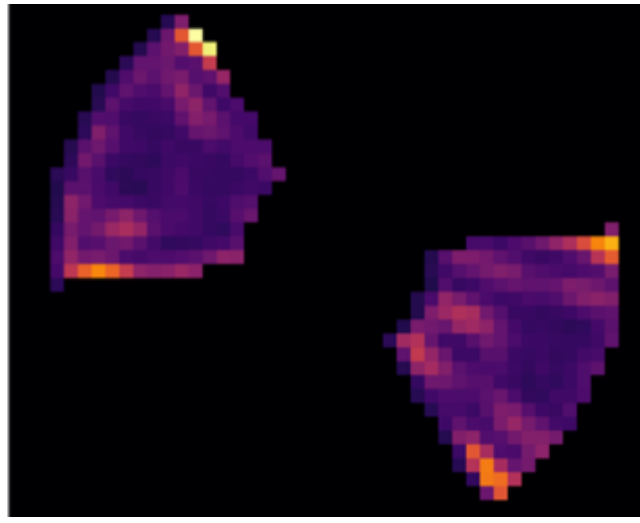

(a) Before RDI

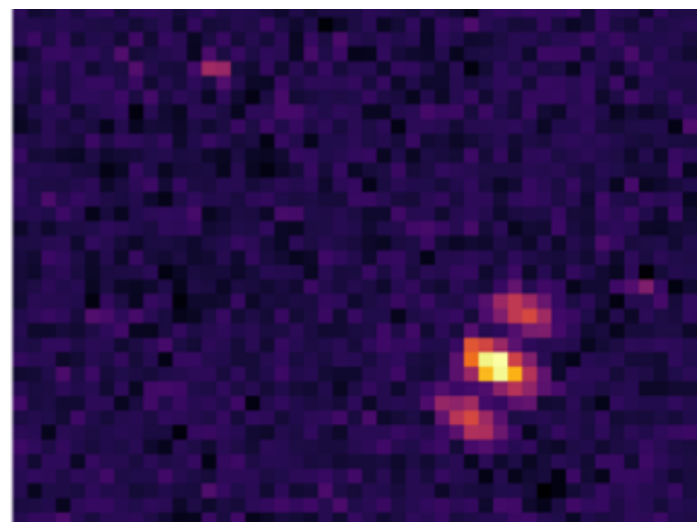

(b) After RDI

Figure 10. (a) Average of target slice for a beginning-of-life detector with no cosmic rays and no hot pixels. (b) Average target slice after removing the best-fit reference slice, where the planet PSF with three lobes is clearly detected in the lower right half of the dark hole.

The differences between steps simulated at different times throughout the detector lifetime are a decrease in detector quantum efficiency and an increase in the number of hot pixels, both effects being modeled after an empirical model using laboratory measurements (Morrissey, private communication) and will continually be refined through Phases B and C.

For these simulations, the post-processing is essential and kept to a minimal amount of complexity - a simple least squares subtraction is applied slice by slice by using the reference star observations. Because the SNR is too low in individual 2-hour averages, we stack all averages together before applying the least squares reference differential algorithm.

A matched filter is used to extract the spectrum from the point source in both cases (Fig. 12), using a forward model of an off-axis PSF at the same location of the planet. The contrast is obtained by normalizing by a simulated observation of the off-axis unocculted star with the IFS. In practice, this can be achieved using 


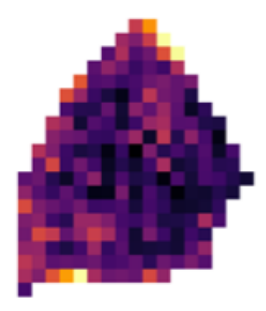

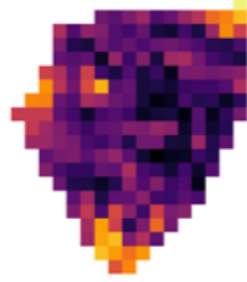

(a) Before RDI

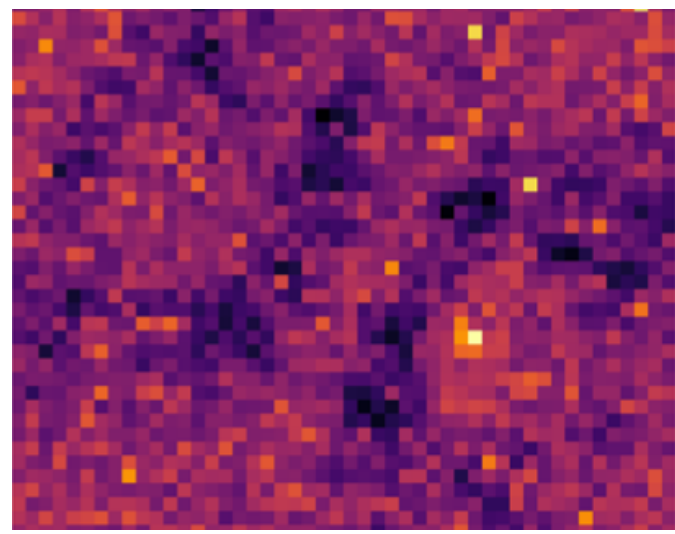

(b) After RDI

Figure 11. (a) Average of target slice for a detector with cosmic rays and hot pixels after 3 years of radiation dosage in the L2 environment. (b) Average target slice after removing the best-fit reference slice. In this case, some over-subtraction is visible, and the planet is barely detected in the lower right half of the dark hole.

a neutral density filter. However, because the detector quantum efficiency degrades as a function of photon flux for very low count rates, some global adjustment is necessary to obtain a properly-calibrated contrast curve. The details of how to do this are still in development.

The final step of the process, which is not yet implemented in this work, is to compute correct error bars. We have developed a formalism to do this optimally from a statistical point of view for a given extraction step, but we still need to propagate errors through our post-processing pipeline to understand what the final errors on the extracted spectrum look like.

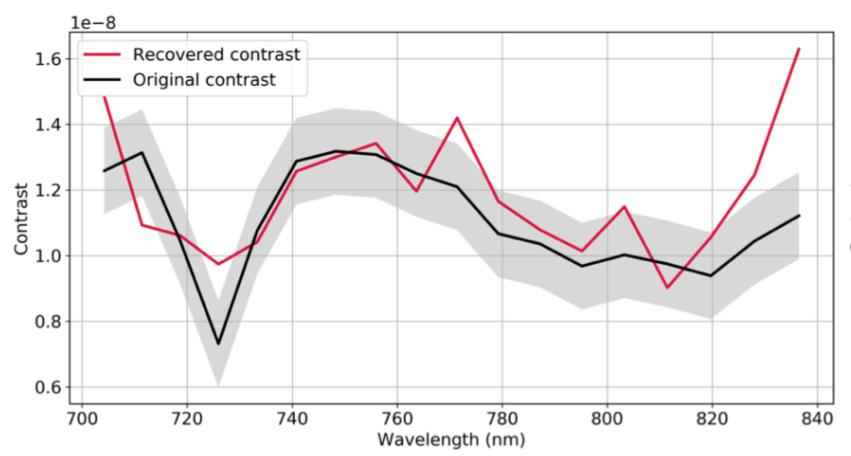

(a) Case 1

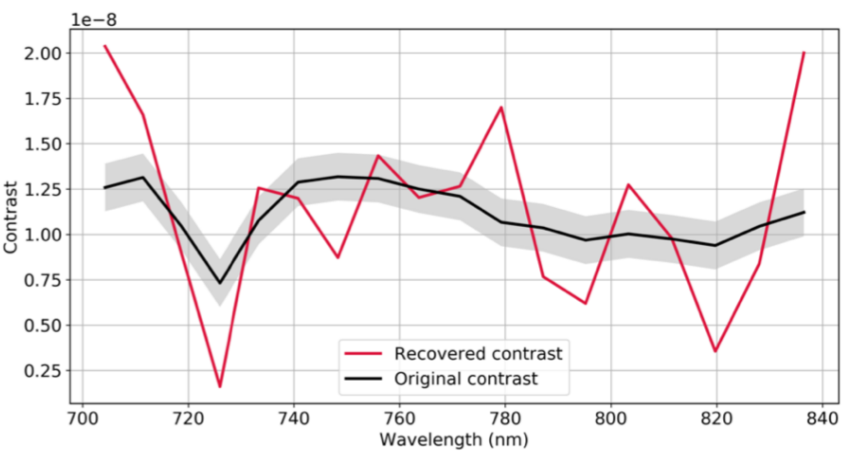

(b) Case 2

Figure 12. (a) Recovered contrast in the case of a beginning of life detector with no cosmic rays or hot pixels. (b) Recovered contrast in the case of a detector with cosmic rays and hot pixels after 3 years of radiation dosage in the L2 environment.

\section{CONCLUSIONS}

We have built up an open-source framework which is able to produce realistic data products from WFIRST CGI. The software ingests resolved maps produced and distributed by JPL which describe the predicted behavior of the coronagraph focal plane in a representative observing scenario. We can simulate the CGI imaging mode by adding an arbitrary science scene and propagating it through the system, then apply a detector noise model. The online package also provides some basic level of post-processing.

In addition, the framework can propagate CGI PSFs through the flight IFS model, and extract detector images back into spatio-spectral cubes to produce realistic data products. The spectrum of targets can be extracted 
and the sensitivity of the instruments to various parameters can be assessed. Future work includes propagating errors through the post-processing steps to add statistically robust error bars on the recovered spectra.

Our preliminary analysis of a single system observed by the IFS indicates that cosmic rays are a major concern and can drastically reduce the sensitivity of the IFS if not treated properly. The tool we developed will allow a robust analysis of the sensitivity of the instrument on both instrumental and environmental parameters, and effort which will continue through the future phases of the project.

\section{ACKNOWLEDGMENTS}

Portions of this research were carried out at the Jet Propulsion Laboratory, California Institute of Technology, under a contract with the National Aeronautics and Space Administration.

\section{REFERENCES}

1. P. Saxena, M. J. Rizzo, C. Mejia Prada, J. Llop Sayson, Q. Gong, E. J. Cady, A. M. Mandell, T. D. Groff, and M. W. McElwain, "Commissioning and performance results of the WFIRST/PISCES integral field spectrograph," in Society of Photo-Optical Instrumentation Engineers (SPIE) Conference Series, Society of Photo-Optical Instrumentation Engineers (SPIE) Conference Series 10400, p. 104001P, Sept. 2017.

2. M. W. McElwain, A. M. Mandell, Q. Gong, J. Llop-Sayson, T. Brandt, V. J. Chambers, B. Grammer, B. Greeley, G. Hilton, M. D. Perrin, K. R. Stapelfeldt, R. Demers, H. Tang, and E. Cady, "PISCES: an integral field spectrograph technology demonstration for the WFIRST coronagraph," in Proceedings of the SPIE, H. A. MacEwen, G. G. Fazio, M. Lystrup, N. Batalha, N. Siegler, and E. C. Tong, eds., pp. 99041A99041A-18, NASA Goddard Space Flight Ctr. (United States), SPIE, July 2016.

3. T. D. Groff, C. Mejia Prada, E. Cady, M. J. Rizzo, A. Mandell, Q. Gong, M. McElwain, N. Zimmerman, P. Saxena, and O. Guyon, "Wavefront control methods for high-contrast integral field spectroscopy," in Society of Photo-Optical Instrumentation Engineers (SPIE) Conference Series, Society of Photo-Optical Instrumentation Engineers (SPIE) Conference Series 10400, p. 104000Q, Sept. 2017.

4. M. J. Rizzo, T. D. Groff, N. T. Zimmermann, Q. Gong, A. M. Mandell, P. Saxena, M. W. McElwain, A. Roberge, J. Krist, A. J. E. Riggs, E. J. Cady, C. Mejia Prada, T. Brandt, E. Douglas, and K. Cahoy, "Simulating the WFIRST coronagraph integral field spectrograph," in Society of Photo-Optical Instrumentation Engineers (SPIE) Conference Series, Society of Photo-Optical Instrumentation Engineers (SPIE) Conference Series 10400, p. 104000B, Sept. 2017.

5. T. D. Brandt, M. Rizzo, T. Groff, J. Chilcote, J. P. Greco, N. J. Kasdin, M. A. Limbach, M. Galvin, C. Loomis, G. Knapp, M. W. McElwain, N. Jovanovic, T. Currie, K. Mede, M. Tamura, N. Takato, and M. Hayashi, "Data Reduction Pipeline for the CHARIS Integral-Field Spectrograph," arXiv.org, June 2017.

6. L. Pueyo, "Detection and characterization of exoplanets using projections on karhunen loeve eigenimages: Forward modeling," The Astrophysical Journal 824(2), p. 117, 2016.

7. A. Roberge, M. J. Rizzo, A. P. Lincowski, G. N. Arney, C. C. Stark, T. D. Robinson, G. F. Snyder, L. Pueyo, N. T. Zimmerman, T. Jansen, E. R. Nesvold, V. S. Meadows, and M. C. Turnbull, "Finding the Needles in the Haystacks: High-fidelity Models of the Modern and Archean Solar System for Simulating Exoplanet Observations," Publications of the Astronomical Society of Pacific 129, pp. 124401-, Dec. 2017.

8. C. C. Stark, "The Transit Light Curve of an Exozodiacal Dust Cloud," 142, p. 123, Oct. 2011. 